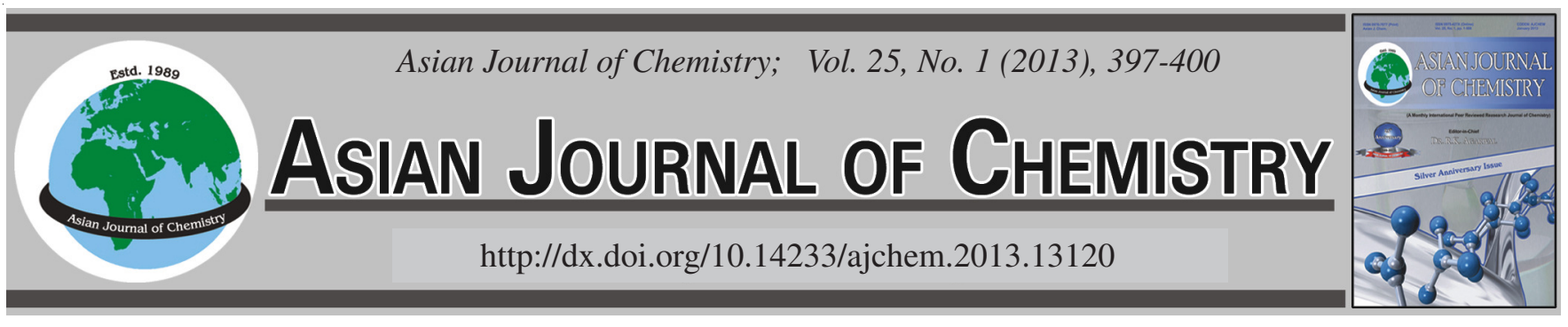

\title{
Synthesis and Antibacterial Activities of Pleuromutilin Derivatives with Modified 7-Aminocephalosporin Acid and Thioether Moiety
}

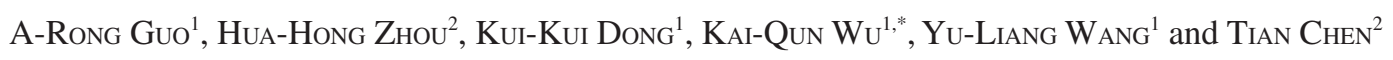

${ }^{1}$ Faculty of Chemistry, Sichuan University, Chengdu 610065, P.R. China

${ }^{2}$ Department of Pathogenic Biology, School of Biomedical Science, Chengdu Medical College, Chengdu 610083, P.R. China

*Corresponding author: Tel: +86 13708058490; E-mail: wukaiqun@tom.com; gyr9.221@163.com

\begin{abstract}
In order to study the effect of heterocyclic carboxamide group on pleuromutilin's antibacterial activity, seven novel pleuromutilin derivatives with modified 7-aminocephalosporin acid and thioether moiety were designed and synthesized. The antibacterial activities of the new compounds were tested via agar-well diffusion method in vitro under different concentrations. The results showed that three target compounds (5a, 5d, 5f) still had antibacterial activity against Staphylococcus aureus SC and Staphylococcus aureus ATCC26112 at the concentration $2.0 \mu \mathrm{g} / \mathrm{mL}$.
\end{abstract}

Key Words: Pleuromutilin derivatives, 7-Aminocephalosporin acid, Synthesis, Antibacterial activity, Agar-well diffusion.

\section{INTRODUCTION}

The antibiotic pleuromutilin (Fig. 1) was first isolated in 1951 by Kavanagh and co-workers ${ }^{1}$. Early research showed that this kind of antibiotic has modest activity in vitro against Gram-positive pathogens and mycoplasmas ${ }^{2,3}$. Pleuromutilin selectively inhibits bacterial protein synthesis through interaction with prokaryotic ribosome ${ }^{4}$. A Sandoz group 5 indicated that the modification at $\mathrm{C} 14$ side chain could be the most promising approach to obtain optimum activity and the combinations of both the thioether group and the basic group in the side chain would give excellent bioactivity. Based on these structure and activity relationships, tiamulin ${ }^{6}$, valunemulin ${ }^{7,8}$ and retapamulin ${ }^{9,10}$ were successfully developed as therapeutic pharmaceuticals. In 2008, pleuromutilin analogs with purine ring possessing excellent antibacterial activity had been reported by Hirokawa and coworkers ${ }^{11}$.
In our previous works, some pleuromutilin derivatives containing substituent pyrazole carboxamide ${ }^{12}$ and substituent thiazole carboxamide ${ }^{13}$ (Fig. 1) were reported to exist excellent antibacterial activity. Herein, for the propose of further exploring the effect of heterocyclic carboxamide groups on the antibacterial activity of pleuromutilin, some modified 7-aminocephalosporin acids (7-ACA) was introduced into pleuromutilin by amido bond. The synthetic route was showed in Scheme-I, the antibacterial activities of the target compounds were tested via agar-well diffusion method.

\section{EXPERIMENTAL}

Compounds 5a-g were prepared as shown in Scheme-I. Intermediates 2a-g were prepared according to the literature with little modification ${ }^{14}$. Intermediates $\mathbf{2}$ was suspended in dried dichloromethane and $\mathrm{Et}_{3} \mathrm{~N}$ at $0{ }^{\circ} \mathrm{C}$ where 1-(3-dimethylaminopropyl)-3-ethylcarbodiimide hydrochloride (EDCI) and

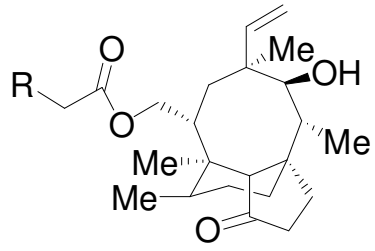

Pleuromutilin(1): $\mathrm{R}=\mathrm{OH}$

Tiamutilin(2): $\mathrm{R}=-\mathrm{S}$
Valunemulin(3): R = S<smiles>CNC(=O)C(N)C(C)C</smiles>

(5): $\mathrm{R}=$<smiles>CCC(C)(C)SC</smiles>

(6): $\mathrm{R}=$<smiles>[R5]c1nn(CC)c(C(N)=O)c1[R5]</smiles>

$\mathrm{R}_{2}$<smiles>[R]c1ncc(C=C)s1</smiles>

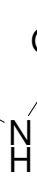

S<smiles>CCNC(C)(C)C</smiles>

Fig. 1. Pleuromutilin and pleuromutilin derivaties 


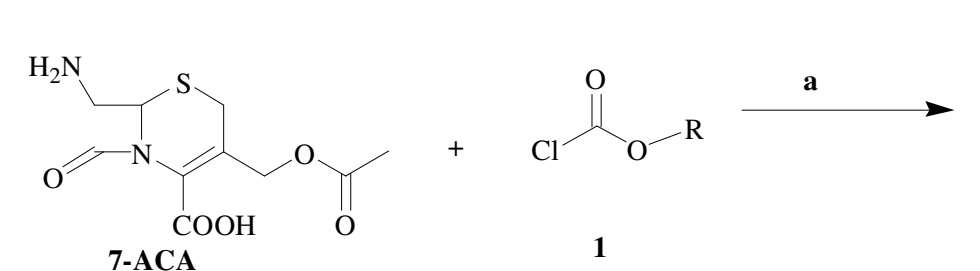<smiles>[R]OC(=O)NC1C(=O)N2C(C(=O)O)=C(COC(C)=O)CSC12</smiles>

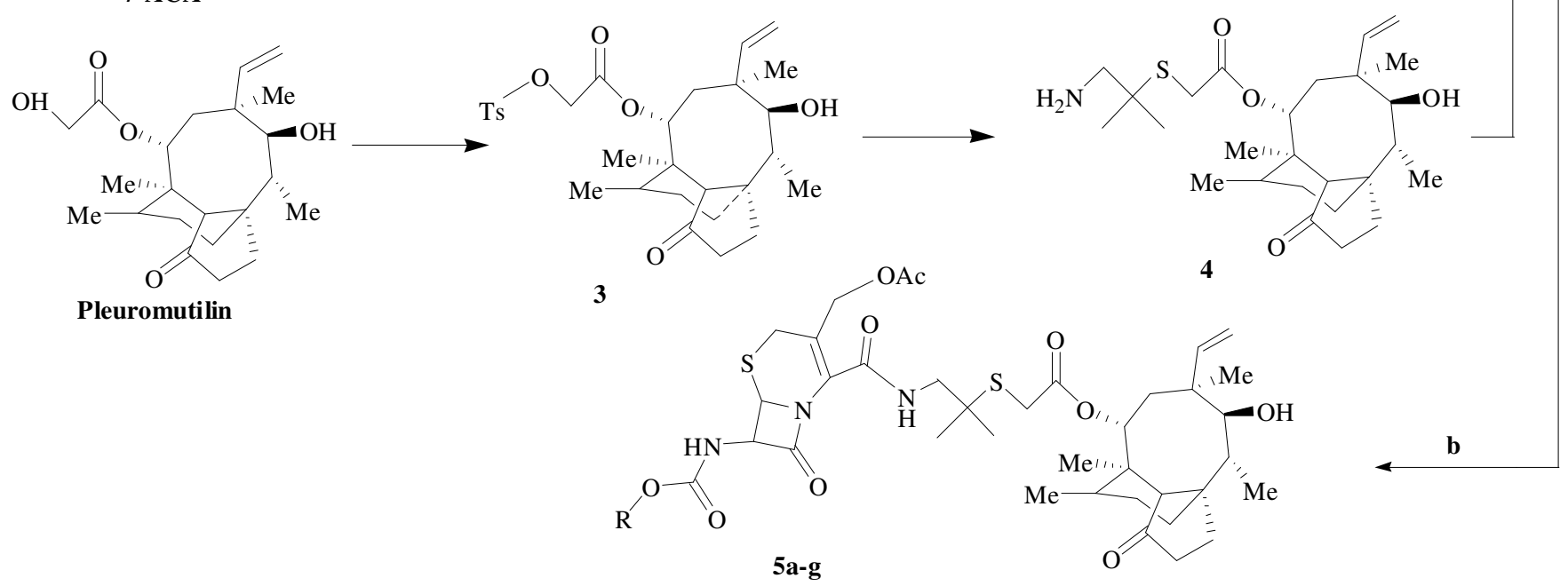

2a,5a: $\mathrm{R}=\mathrm{CH}_{3} ; \mathbf{2 b , 5 b : ~} \mathrm{R}=\mathrm{CH}_{3} \mathrm{CH}_{2} ; \mathbf{2 c}, \mathbf{5 c}: \mathrm{R}=\left(\mathrm{CH}_{3}\right)_{2} \mathrm{CH} ; \mathbf{2 d}, \mathbf{5 d}: \mathrm{R}=\left(\mathrm{CH}_{3}\right)_{2} \mathrm{CH}_{2} \mathrm{CH} ; \mathbf{2 e}, \mathbf{5 e}: \mathrm{R}=\left(\mathrm{CH}_{3}\right)_{3} \mathrm{C} ; \mathbf{2 f}, \mathbf{5 f}: \mathrm{R}=\mathrm{C}_{6} \mathrm{H}_{5} \mathrm{CH}_{2} ; \mathbf{2 g}, \mathbf{5 g}: \mathrm{R}=\mathrm{CCl}_{3} \mathrm{CH}_{2}$

Scheme-I: Synthetic route of compound $\mathbf{5 a - g}$

1-hydroxybenzotriazole (HOBT) were rapidly added and the reaction mixture was maintained at room temperature for $2 \mathrm{~h}$. Then compound $\mathbf{4}$ (which was synthesized according to the literature ${ }^{12}$ ) was added into the solution at $0{ }^{\circ} \mathrm{C}$ and the resulting solution was allowed to be stirred at room temperature for another $24 \mathrm{~h}$. The solution was then washed with $\mathrm{H}_{2} \mathrm{O}$ and saturated sodium chloride solution. The organic layer was dried, filtered and evaporated under reduced pressure. The crude products were purified on silica-gel column (petroleum ether/ethyl acetate as the eluent).

Mutilin-14-0-[1-(7-methoxycarbamidocephalosporanic carboxamide)-2-methylpropane-2-yl] thioacetate (5a): Orange solid; yield: $76.34 \%$; m.p.: 106-108 ${ }^{\circ} \mathrm{C}$; IR (KBr, $\left.v_{\max }, \mathrm{cm}^{-1}\right): 3402,3084,2931,2870,1729,1664,1537,1459$, $1375,1280,1117,1022,915,587 ;{ }^{1} \mathrm{H}$ NMR spectrum $(400$ MHz; d1-CDCl 3 ; TMS): $\delta$ (ppm) $0.73(\mathrm{~d}, 3 \mathrm{H}, J=6.8 \mathrm{~Hz})$, $0.90(\mathrm{~d}, 3 \mathrm{H}, J=6.8 \mathrm{~Hz}), 1.10-1.15(\mathrm{~m}, 1 \mathrm{H}), 1.18(\mathrm{~s}, 3 \mathrm{H})$, $1.26-1.28(\mathrm{~m}, 3 \mathrm{H}), 1.31(\mathrm{~s}, 3 \mathrm{H}), 1.33-1.45(\mathrm{~m}, 3 \mathrm{H}), 1.47$ (s, $3 \mathrm{H}), 1.60$ (s, 3H), 1.65-1.71 (m, 3H), 1.77-1.81 (m, 1H), 2.06 $(\mathrm{s}, 3 \mathrm{H}), 2.07-2.14(\mathrm{~m}, 3 \mathrm{H}), 2.18-2.35(\mathrm{~m}, 3 \mathrm{H}), 3.12-3.30(\mathrm{~m}$, $6 \mathrm{H}), 3.32-3.38(\mathrm{~m}, 2 \mathrm{H}), 3.73(\mathrm{~s}, 3 \mathrm{H}), 5.19-5.23(\mathrm{~m}, 2 \mathrm{H}), 5.32$ $\left(\mathrm{dd}, 1 \mathrm{H}, J_{1}=10.8 \mathrm{~Hz}, J_{2}=1.2 \mathrm{~Hz}\right), 5.74-5.78(\mathrm{~m}, 2 \mathrm{H}), 6.48$ (dd, $\left.1 \mathrm{H}, J_{1}=17.6 \mathrm{~Hz}, J_{2}=11.2 \mathrm{~Hz}\right)$; HR-MS (-ESI): Calcd. for $\mathrm{C}_{38} \mathrm{H}_{54} \mathrm{~N}_{3} \mathrm{O}_{10} \mathrm{~S}_{2}[\mathrm{M}-\mathrm{H}]^{-}:$776.3251, Found: 776.3265 .

Mutilin-14-O-[1-(7-ethoxycarbamidocephalosporanic carboxamide)-2-methylpropane-2-yl] thioacetate (5b): Yellow solid; yield: $75.83 \%$; m.p.: $94-96{ }^{\circ} \mathrm{C}$; IR (KBr, $v_{\max }$, $\left.\mathrm{cm}^{-1}\right): 3393,3075,2960,2929,2857,1775,1726,1677,1537$, $1457,1377,1262,1115,1022,914,802,731 ;{ }^{1} \mathrm{H}$ NMR spectrum (400 MHz; d1-CDCl ${ }_{3}$; TMS): $\delta$ (ppm) 0.67 (d, 3H, $J$ $=6.8 \mathrm{~Hz}), 0.85(\mathrm{~d}, 3 \mathrm{H}, J=6.8 \mathrm{~Hz}), 1.02-1.08(\mathrm{~m}, 1 \mathrm{H}), 1.12$ $(\mathrm{m}, 3 \mathrm{H}), 1.18(\mathrm{~s}, 3 \mathrm{H}), 1.19(\mathrm{~s}, 3 \mathrm{H}), 1.26-1.37(\mathrm{~m}, 3 \mathrm{H}), 1.40(\mathrm{~s}$, $3 \mathrm{H}), 1.43-1.57$ (m, 3H), 1.65-1.77 (m, 1H), 1.99 (s, 3H, 2.02-
2.09 (m, 3H), 2.12-2.33 (m, 3H), 3.06-3.18 (m, 3H), 3.203.29 (m, 3H), 3.31-3.34 (m, 2H), $3.65(\mathrm{~m}, 2 \mathrm{H}), 4.61(\mathrm{~d}, 1 \mathrm{H}$, $J=12.8 \mathrm{~Hz}), 4.84(\mathrm{~d}, 1 \mathrm{H}, J=12.8 \mathrm{~Hz}), 4.90(\mathrm{~s}, 1 \mathrm{H}), 5.14(\mathrm{dd}$, $\left.1 \mathrm{H}, J_{1}=17.2 \mathrm{~Hz}, J_{2}=9.2 \mathrm{~Hz}\right), 5.23\left(\mathrm{dd}, 1 \mathrm{H}, J_{1}=10.4 \mathrm{~Hz}, J_{2}\right.$ $=8.4 \mathrm{~Hz}), 5.40(\mathrm{~m}, 1 \mathrm{H}), 5.48-5.51(\mathrm{~m}, 1 \mathrm{H}), 6.56(\mathrm{~s}, 1 \mathrm{H}), 7.60$ (s, 1H); HR-MS (-ESI): Calcd. for $\mathrm{C}_{39} \mathrm{H}_{56} \mathrm{~N}_{3} \mathrm{O}_{10} \mathrm{~S}_{2}[\mathrm{M}-\mathrm{H}]^{-}$: 790.3406, Found: 790.3412.

Mutilin-14-O-[1-(7-isopropoxycarbamidocephalosporanic carboxamide)-2-methylpropane-2-yl] thioacetate (5c): Yellow solid; yield: $61.28 \%$; m.p.: $98-102{ }^{\circ} \mathrm{C}$; IR (KBr, $\left.v_{\max }, \mathrm{cm}^{-1}\right): 3441,2961,2927,2857,1778,1726,1532,1459$, 1379, 1260, 1111, 1024, 914, 803, 616; ${ }^{1} \mathrm{H}$ NMR spectrum (400 MHz; d1-CDCl ${ }_{3}$; TMS): $\delta$ (ppm) 0.73 (d, 3H, $J=6.8$ $\mathrm{Hz}), 0.90$ (d, 3H, J=7.2 Hz), 1.11-1.14 (m, 1H), 1.17 (s, 3H), 1.22-1.28 (m, 6H), $1.30(\mathrm{~s}, 3 \mathrm{H}), 1.32-1.42(\mathrm{~m}, 3 \mathrm{H}), 1.46(\mathrm{~s}$, $3 \mathrm{H}), 1.56(\mathrm{~s}, 3 \mathrm{H}), 1.62-1.70(\mathrm{~m}, 3 \mathrm{H}), 1.80-1.83(\mathrm{~m}, 1 \mathrm{H}), 2.05$ (s, 3H), 2.10-2.16 (m, 3H), 2.17-2.44 (m, 3H), 3.14-3.30 (m, $6 \mathrm{H}), 3.34-3.56(\mathrm{~m}, 2 \mathrm{H}), 4.69$ (d, 1H, $J=12.8 \mathrm{~Hz}), 4.90-5.03$ $(\mathrm{m}, 2 \mathrm{H}), 5.20\left(\mathrm{dd}, 1 \mathrm{H}, J_{1}=17.6 \mathrm{~Hz}, J_{2}=9.6 \mathrm{~Hz}\right), 5.31(\mathrm{~d}, 1 \mathrm{H}$, $J=7.6 \mathrm{~Hz}), 5.36-5.49(\mathrm{~m}, 1 \mathrm{H}), 5.80(\mathrm{~d}, 1 \mathrm{H}, J=8.8 \mathrm{~Hz}), 6.44$ $\left(\mathrm{dd}, 1 \mathrm{H}, J_{1}=17.2 \mathrm{~Hz}, J_{2}=11.2 \mathrm{~Hz}\right), 7.52(\mathrm{~s}, 1 \mathrm{H}), 7.56(\mathrm{~s}, 1 \mathrm{H})$; HR-MS (-ESI): Calcd. for $\mathrm{C}_{40} \mathrm{H}_{58} \mathrm{~N}_{3} \mathrm{O}_{10} \mathrm{~S}_{2}[\mathrm{M}-\mathrm{H}]^{-}:$: 804.3563, Found: 804.3567.

Mutilin-14-O-[1-(7-isobutoxycarbamidocephalosporanic carboxamide)-2-methylpropane-2-yl] thioacetate (5d): Yellow solid; yield: $63.57 \%$; m.p.: $71-73{ }^{\circ} \mathrm{C}$; IR (KBr, $\left.V_{\max }, \mathrm{cm}^{-1}\right): 3403,3079,2921,2928,2865,1729,1663,1540$, 1461, 1375, 1280, 1117, 1023, 915; ${ }^{1} \mathrm{H}$ NMR spectrum (400 MHz; d1-CDCl ${ }_{3}$; TMS): $\delta$ (ppm) 0.73 (d, 3H, $J=7.2 \mathrm{~Hz}$ ), $0.90(\mathrm{~d}, 3 \mathrm{H}, J=6.8 \mathrm{~Hz}), 0.99(\mathrm{~d}, 6 \mathrm{H}, J=6.8 \mathrm{~Hz}), 1.10-1.15$ $(\mathrm{m}, 1 \mathrm{H}), 1.18(\mathrm{~s}, 3 \mathrm{H}), 1.21-1.28(\mathrm{~m}, 3 \mathrm{H}), 1.31(\mathrm{~s}, 3 \mathrm{H}), 1.35-$ $1.43(\mathrm{~m}, 3 \mathrm{H}), 1.46(\mathrm{~s}, 3 \mathrm{H}), 1.48-1.62(\mathrm{~m}, 3 \mathrm{H}), 1.65-1.70(\mathrm{~m}$, $3 \mathrm{H}), 1.76-1.80$ (m, 1H), 1.99 (s, 3H), 2.05-2.18 (m, 3H), 2.20- 
$2.35(\mathrm{~m}, 4 \mathrm{H}), 3.11-3.31(\mathrm{~m}, 6 \mathrm{H}), 3.36-3.40(\mathrm{~m}, 2 \mathrm{H}), 4.09(\mathrm{~d}$, $2 \mathrm{H}, J=6.8 \mathrm{~Hz}), 5.27\left(\mathrm{dd}, 1 \mathrm{H}, J_{1}=17.6 \mathrm{~Hz}, J_{2}=1.6 \mathrm{~Hz}\right), 5.32$ $\left(\mathrm{dd}, 1 \mathrm{H}, J_{1}=11.2 \mathrm{~Hz}, J_{2}=1.6 \mathrm{~Hz}\right), 5.74(\mathrm{~d}, 1 \mathrm{H}, J=8.4 \mathrm{~Hz})$, $6.43\left(\mathrm{dd}, 1 \mathrm{H}, J_{1}=17.2 \mathrm{~Hz}, J_{2}=10.8 \mathrm{~Hz}\right), 7.52(\mathrm{~m}, 1 \mathrm{H})$; HRMS (-ESI): Calcd. for $\mathrm{C}_{41} \mathrm{H}_{60} \mathrm{~N}_{3} \mathrm{O}_{10} \mathrm{~S}_{2}[\mathrm{M}-\mathrm{H}]^{-}:$: 818.3719, Found: 818.3725.

Mutilin-14-O-[1-(7-t-butoxycarbamidocephalosporanic carboxamide)-2-methylpropane-2-yl] thioacetate (5e): Yellow solid; yield: $56.78 \%$; m.p.: $86-88^{\circ} \mathrm{C}$; IR (KBr, $\left.v_{\max }, \mathrm{cm}^{-1}\right): 3419,2959,2924,2855,1726,1526,1459,1372$, $1261,1110,1023,914,803,618,588 ;{ }^{1} \mathrm{H}$ NMR spectrum (400 $\mathrm{MHz}$; d1-CDCl ${ }_{3}$; TMS): $\delta$ (ppm) $0.73(\mathrm{~d}, 3 \mathrm{H}, J=6.8 \mathrm{~Hz})$, $0.88(\mathrm{~d}, 3 \mathrm{H}, J=6.8 \mathrm{~Hz}), 1.10-1.14(\mathrm{~m}, 1 \mathrm{H}), 1.17(\mathrm{~s}, 3 \mathrm{H})$, $1.21-1.24(\mathrm{~m}, 3 \mathrm{H}), 1.25(\mathrm{~s}, 9 \mathrm{H}), 1.28(\mathrm{~s}, 3 \mathrm{H}), 1.36-1.39(\mathrm{~m}$, $3 \mathrm{H}), 1.45(\mathrm{~s}, 3 \mathrm{H}), 1.48-1.69(\mathrm{~m}, 3 \mathrm{H}), 2.05(\mathrm{~s}, 3 \mathrm{H}), 2.10(\mathrm{~s}$, $3 \mathrm{H}), 2.17-2.33(\mathrm{~m}, 3 \mathrm{H}), 3.12-3.31(\mathrm{~m}, 6 \mathrm{H}), 3.33-3.80(\mathrm{~m}, 2 \mathrm{H})$, 4.67-4.79 (m, 2H), 4.96 (d, 1H, $J=11.6 \mathrm{~Hz}), 5.19$ (dd, 1H, $J_{1}$ $\left.=17.6 \mathrm{~Hz}, J_{2}=8.0 \mathrm{~Hz}\right), 5.47(\mathrm{~d}, 1 \mathrm{H}, J=8.4 \mathrm{~Hz}), 5.55(\mathrm{~m}$, $1 \mathrm{H}), 5.76(\mathrm{~d}, 1 \mathrm{H}, J=8.4 \mathrm{~Hz}), 6.45\left(\mathrm{dd}, 1 \mathrm{H}, J_{1}=16.4 \mathrm{~Hz}, J_{2}=\right.$ $6.0 \mathrm{~Hz}$ ); HR-MS (-ESI): Calcd. for $\mathrm{C}_{41} \mathrm{H}_{60} \mathrm{~N}_{3} \mathrm{O}_{10} \mathrm{~S}_{2}[\mathrm{M}-\mathrm{H}]^{-}$: 818.3719, Found: 818.3728.

Mutilin-14-0-[1-(7-benzoxycarbamidocephalosporanic carboxamide)-2-methylpropane-2-yl] thioacetate (5f): Yellow solid; yield: $64.66 \%$; m.p.: $58-60{ }^{\circ} \mathrm{C}$; IR ( $\mathrm{KBr}$, $\left.V_{\max }, \mathrm{cm}^{-1}\right): 3388,2962,2924,2855,1729,1661,1524,1458$, $1377,1261,1097,1022,912,800,698,586 ;{ }^{1} \mathrm{H}$ NMR spectrum (400 MHz; d1-CDCl 3 ; TMS): $\delta$ (ppm) 0.73 (d, 3H, $J=$ $6.8 \mathrm{~Hz}), 0.90(\mathrm{~d}, 3 \mathrm{H}, J=6.8 \mathrm{~Hz}), 1.10-1.15(\mathrm{~m}, 1 \mathrm{H}), 1.18$ (s, $3 \mathrm{H}), 1.26-1.28$ (m, 3H), 1.31 (s, 3H), 1.36-1.43 (m, 3H), 1.46 $(\mathrm{s}, 3 \mathrm{H}), 1.49-1.62(\mathrm{~m}, 3 \mathrm{H}), 1.65-1.70(\mathrm{~m}, 3 \mathrm{H}), 1.76-1.80(\mathrm{~m}$, $1 \mathrm{H}), 2.02$ (s, 3H), 2.05-2.13 (m, 3H), 2.20-2.35 (m, 3H), 3.11$3.27(\mathrm{~m}, 6 \mathrm{H}), 3.29-3.37(\mathrm{~m}, 2 \mathrm{H}), 5.21\left(\mathrm{dd}, 2 \mathrm{H}, J_{1}=17.2 \mathrm{~Hz}\right.$, $\left.J_{2}=1.2 \mathrm{~Hz}\right), 5.33(\mathrm{~d}, 2 \mathrm{H}, J=12.0 \mathrm{~Hz}), 5.75(\mathrm{~d}, 1 \mathrm{H}, J=8.8$ $\mathrm{Hz}), 6.48\left(\mathrm{dd}, 1 \mathrm{H}, J_{1}=17.6 \mathrm{~Hz}, J_{2}=11.2 \mathrm{~Hz}\right), 7.34(\mathrm{~m}, 5 \mathrm{H})$; HR-MS (-ESI): Calcd. for $\mathrm{C}_{44} \mathrm{H}_{58} \mathrm{~N}_{3} \mathrm{O}_{10} \mathrm{~S}_{2}[\mathrm{M}-\mathrm{H}]^{-}:$: 852.3563, Found: 852.3581.

Mutilin-14-O-[1-(7-N-carbamate-2,2,2-trichloroethyl) -cephalosporanic carboxamide)-2-methylpropane-2-yl] thioacetate (5g): Yellow solid; yield: 57.89 \%; m.p.: 68-70 ${ }^{\circ} \mathrm{C}$; IR $\left(\mathrm{KBr}, \mathrm{v}_{\max }, \mathrm{cm}^{-1}\right): 3347,2962,2926,2858,1733,1679$, 1533, 1457, 1380, 1261, 1101, 1020, 866, 799, 702, 570; ${ }^{1} \mathrm{H}$ NMR spectrum (400 MHz; d1-CDCl $;$; TMS): $\delta$ (ppm) 0.74 $(\mathrm{d}, 3 \mathrm{H}, J=6.8 \mathrm{~Hz}), 0.88(\mathrm{~d}, 3 \mathrm{H}, J=6.8 \mathrm{~Hz}), 1.10-1.15(\mathrm{~m}$, $1 \mathrm{H}), 1.17(\mathrm{~s}, 3 \mathrm{H}), 1.31(\mathrm{~s}, 3 \mathrm{H}), 1.33(\mathrm{~s}, 3 \mathrm{H}), 1.37-1.43(\mathrm{~m}$, $3 \mathrm{H}), 1.47$ (s, 3H), 1.49-1.58 (m, 3H), 1.61-1.70 (m, 3H), 1.76$1.80(\mathrm{~m}, 1 \mathrm{H}), 2.02(\mathrm{~s}, 3 \mathrm{H}), 2.05-2.20(\mathrm{~m}, 3 \mathrm{H}), 2.22-2.35(\mathrm{~m}$, $3 \mathrm{H}), 3.16-3.30$ (m, 6H), 3.35 (d, $1 \mathrm{H}, J=6.4 \mathrm{~Hz}), 3.48$ (dd, $1 \mathrm{H}$, $\left.J_{1}=14.1 \mathrm{~Hz}, J_{2}=6.8 \mathrm{~Hz}\right), 5.17(\mathrm{~d}, 1 \mathrm{H}, J=17.5 \mathrm{~Hz}), 5.26(\mathrm{~d}$, $1 \mathrm{H}, J=11.12 \mathrm{~Hz}), 5.77(\mathrm{~d}, 1 \mathrm{H}, J=8.5 \mathrm{~Hz}), 6.47\left(\mathrm{dd}, 1 \mathrm{H}, J_{1}=\right.$ $\left.17.6 \mathrm{~Hz}, J_{2}=11.2 \mathrm{~Hz}\right), 7.67(\mathrm{~s}, 1 \mathrm{H}), 7.89$ (d, 1H, $\left.J=8.0 \mathrm{~Hz}\right)$; HR-MS (-ESI): Calcd. for $\mathrm{C}_{39} \mathrm{H}_{53} \mathrm{~N}_{3} \mathrm{O}_{10} \mathrm{~S}_{2} \mathrm{Cl}_{3}[\mathrm{M}-\mathrm{H}]^{-}$: 892.2237, 894.2208. Found: $892.2247,894.2225$ [isotope peaks].

The antibacterial activities of the target compounds were tested via agar-well diffusion method in vitro. Target compound $(1000 \mu \mathrm{g})$ was dissolved with ethanol $(1 \mathrm{~mL})$ and diluted to $20 \mu \mathrm{g} / \mathrm{mL}$ with ethanol, later, 5a, $5 \mathbf{d}$ and $\mathbf{5 f}$ were further diluted to $2 \mu \mathrm{g} / \mathrm{mL}$. A $50 \mu \mathrm{L}$ solution of each compound (target compounds or pleuromutilin) was injected into the corresponding well in the Luria Bertani (LB) culture medium, which was covered with bacteria suspension in advance and the plates were incubated at $37{ }^{\circ} \mathrm{C}$ for $24 \mathrm{~h}$. The results of average diameters of the bacteriostatic circle were listed in Tables 1 and 2.

\begin{tabular}{|c|c|c|}
\hline \multicolumn{3}{|c|}{$\begin{array}{c}\text { TABLE-1 } \\
\text { In vitro ANTIBACTERIAL ACTIVITY OF 5a-g AT } \\
\text { THE CONCENTRATION } 20 \mu \mathrm{g} / \mathrm{mL}\end{array}$} \\
\hline \multirow[b]{2}{*}{ Compound } & \multicolumn{2}{|c|}{ Diameter of inhibition zone (mm) } \\
\hline & $\begin{array}{c}\text { Staphylococcus } \\
\text { aureus SC }\end{array}$ & $\begin{array}{c}\text { Staphylococcus aureus } \\
\text { ATCC } 26112\end{array}$ \\
\hline $5 \mathbf{a}$ & 21 & 17 \\
\hline $5 \mathbf{b}$ & 20 & 15 \\
\hline $5 c$ & 14 & 12 \\
\hline $5 d$ & 20 & 16 \\
\hline $5 e$ & 15 & 12 \\
\hline $5 f$ & 24 & 19 \\
\hline $5 \mathrm{~g}$ & 15 & 13 \\
\hline Pleuromutilin $^{\mathrm{a}}$ & 22 & 13 \\
\hline Ethanol $^{\mathrm{b}}$ & - & - \\
\hline
\end{tabular}

${ }^{\mathrm{a}}$ Concentration of pleuromutilin: $20.0 \mu \mathrm{g} / \mathrm{mL}$; as the positive control; ${ }^{b}$ Negative control: ethanol; Diameter of the well in each plate: $6 \mathrm{~mm}$

\begin{tabular}{|c|c|c|}
\hline \multicolumn{3}{|c|}{$\begin{array}{c}\text { TABLE-2 } \\
\text { In vitro ANTIBACTERIAL ACTIVITY OF 5a, 5d and } \mathbf{5 f} \\
\text { AT THE CONCENTRATION } 2 \mu \mathrm{g} / \mathrm{mL}\end{array}$} \\
\hline \multirow[b]{2}{*}{ Compound } & \multicolumn{2}{|c|}{ Diameter of inhibition zone (mm) } \\
\hline & $\begin{array}{c}\text { Staphylococcus } \\
\text { aureus SC }\end{array}$ & $\begin{array}{c}\text { Staphylococcus } \\
\text { aureus ATCC26112 } \\
\end{array}$ \\
\hline $5 \mathbf{a}$ & 10 & 7 \\
\hline 5d & 11 & 7 \\
\hline $5 f$ & 14 & 11 \\
\hline Pleuromutilin $^{\mathrm{a}}$ & 7 & - \\
\hline Ethanol $^{\mathrm{b}}$ & - & - \\
\hline
\end{tabular}

${ }^{\mathrm{a}}$ Concentration of pleuromutilin: $2.0 \mu \mathrm{g} / \mathrm{mL}$; as the positive control; ${ }^{b}$ Negative control: ethanol; Diameter of the well in each plate: $6 \mathrm{~mm}$

\section{RESULTS AND DISCUSSION}

The results of antibacterial activities showed that all the target compounds exhibited antibacterial activity against two bacterial strains at the concentration $20 \mu \mathrm{g} / \mathrm{mL}$. Three compounds $(\mathbf{5 a}, \mathbf{5 d}, \mathbf{5 f})$ were chosen to further test the antibacterial activity at the concentration $2 \mu \mathrm{g} / \mathrm{mL}$, they still displayed antibacterial activity against Staphylococcus aureus SC and Staphylococcus aureus ATCC26112.

\section{Conclusion}

In conclusion, seven novel pleuromutilin derivatives with modified 7-aminocephalosporin acid and thioether moiety in the $\mathrm{C} 14$ side chain were successfully synthesized. The results of antibacterial activities indicated that three target compounds (5a, 5d, 5f) still exist antibacterial activity against Staphylococcus aureus SC and Staphylococcus aureus ATCC26112 at the concentration $2.0 \mu \mathrm{g} / \mathrm{mL}$. Our exploration has further enriched the content of SAR of pleuromutilin and it is helpful for us to design more pleuromutilin derivatives.

\section{ACKNOWLEDGEMENTS}

The authors appreciated the financial support from the National Science Foundation of China (No. 21072135), the antibacterial activity test of Chengdu Medical College (cx 
20100037) and the ${ }^{1} \mathrm{H}$ NMR analysis by Sichuan University Analytical and Testing Center.

\section{REFERENCES}

1. F. Kavanagh, A. Hervey and W.J. Robbins, Proc. Nat. Acad. Sci. U.S.A., 37, 570 (1951)

2. F. Schlunzen, E. Pyetan and P. Fucini, A. Yonath and J.M. Harms, Mol. Microbial., 5, 1287 (2004).

3. M. Anchel, J. Biol. Chem., 199, 133 (1952).

4. G. Hogenauer, Eur. J. Biochem., 52, 93 (1975).

5. H. Egger and H. Reinshagen, J. Antibiot., 29, 923 (1976).

6. D.G. Burch, G.T. Jones, T.W. Heard and R.E. Tuck, Vet. Rec., 119, 108 (1986).

7. J. Drews, A. Georgopoulos, G. Laber, E. Schütze and J. Unger, Antimicrob. Agents Chemother., 7, 507 (1975).
8. L. Lolk, J. Pøhlsgaard, A.S. Jepsen, L.H. Hansen, H. Nielsen, S.I. Steffansen, L. Sparving, A.B. Nielsen, B. Vester and P. Nielsen, J. Med. Chem., 51, 4957 (2008).

9. L. Parish, A. Free and T. Sumathy, J. Am. Acad. Dermatol., 2, AB11 (2007).

10. Y. Hirokawa, H. Kinoshita, T. Tanaka, K. Nakata, N. Kitadai, K. Fujimoto, S. Kashimoto, T. Kojima and S. Kato, J. Med. Chem., 51, 1991 (2008).

11. Y. Hirokawa, H. Kinoshita, T. Tanaka, T. Nakamura, K. Fujimoto, S. Kashimoto, T. Kojima and S. Kato, Bioorg. Med. Chem., Lett., 18, 3556 (2008).

12. P. Xu, Y.-Y. Zhang, Y.-X. Sun, J.H. Liu, B. Yang, Y.Z. Wang and Y.L. Wang, Chem. Biol. Drug. Des., 73, 655 (2009).

13. L. Wang, F.-Y. Dai J. Zhu, K.-K. Dong, Y.-L. Wang and T. Chen, J. Chem. Res., 35, 313 (2011)

14. R.A. Archer and B.S. Kitchell, J. Org. Chem., 10, 3409 (1966). 